\title{
Pulmonary artery sarcoma diagnosed by endobronchial ultrasound-guided transbronchial needle aspiration
}

\author{
Johnny WM Chan *, Stephanie YY Chu, Connie HK Lam, WH O, OY Cheung, TL Kwan, \\ Alex KC Leung, WL Law
}

\begin{abstract}
A B S T R A C T
Pulmonary artery sarcoma is a rare disease with poor prognosis that has not been reported in Hong Kong. Its clinical and radiological presentation frequently mimics pulmonary embolism. Diagnosis is usually delayed until surgery, which is the treatment option that provides the best survival. Endobronchial ultrasound-guided transbronchial needle aspiration is an effective non-surgical technique for lymph node staging of lung cancer and diagnosis of mediastinal lesions via bronchoscopy. Here we discuss a case of pulmonary artery sarcoma diagnosed by this method, the second one in the literature, which serves to illustrate its potential use for early and minimally invasive diagnosis of the condition. Although such aspiration is a safe procedure, tissue sampling of extravascular extensions is advisable wherever possible.
\end{abstract}

\section{Case report}

A 66-year-old non-smoker Chinese female was hospitalised after her first episode of haemoptysis (approximate volume, $100 \mathrm{~mL}$ ) in February 2012. She reported being in good health, except for an episode of right lower lobe pneumonia about 6 months before presentation, which was treated with antibiotics. Despite radiological recovery, she complained of occasional dry cough, malaise, and weight loss of approximately 10 pounds in the subsequent months.

At admission, she was afebrile, without dyspnoea, and with unremarkable physical findings. Apart from mild anaemia (haemoglobin, $107 \mathrm{~g} / \mathrm{L}$ ) and slightly elevated erythrocyte sedimentation rate of $47 \mathrm{~mm} / \mathrm{h}$, other laboratory tests including white cell and platelet counts, C-reactive protein, coagulation profile, and liver and renal function tests were within normal limits. Culture, acidfast staining, and cytological examination of the sputum did not reveal any abnormality. Radiological examination of the chest revealed a peripheral shadow measuring $1.5 \mathrm{~cm}$ in diameter in the right middle zone. Thoracic computed tomography (CT) revealed a huge contrast-enhancing mass with low, non-homogeneous attenuation filling the right pulmonary artery (PA), extending up to the right
Hong Kong Med J 2014;20:152-5

DOI: 10.12809/hkmj133942

1 JWM Chan *, FRCP, FHKAM (Medicine)

${ }^{1}$ SYY Chu, MRCP, FHKAM (Medicine)

${ }^{1}$ CHK Lam, MRCP, FHKAM (Medicine)

${ }^{1}$ WH O, MRCP, FHKAM (Medicine)

${ }^{2}$ OY Cheung, FRCPath, FHKAM (Pathology)

${ }^{3}$ TL Kwan, FRCR, FHKAM (Radiology)

${ }^{4}$ AKC Leung, FRCR, FHKAM (Radiology)

${ }^{1}$ WL Law, MRCP, FHKAM (Medicine)

1 Department of Medicine

2 Department of Pathology

${ }^{3}$ Department of Radiology and Imaging

${ }^{4}$ Department of Clinical Oncology

Queen Elizabeth Hospital, Jordan, Hong Kong

* Corresponding author: chanwmj@ha.org.hk upper and lower segmental pulmonary arteries, as well as into the mediastinum. Patchy foci were also noted in the pulmonary trunk (Fig 1). Peripheral soft tissue densities were noted in the right middle lobe, which may have been either infarcts or tumour deposits. Bronchoscopy and endobronchial ultrasound-guided transbronchial needle aspiration (EBUS-TBNA) were performed to examine the airways and to obtain histological samples from the mediastinal extension of the mass. No bleeding source or significant endobronchial lesions were identified during bronchoscopy. A heterogeneous mass with distinct margins extending from the lower end of trachea to the right hilar regions along the right main bronchus was revealed by EBUS. Endobronchial ultrasound-guided transbronchial needle aspiration of the mass was performed at the lower end of trachea (Fig 2a). Histological examination revealed tumour fragments with spindle cells lying in myxoid stroma with no definite differentiation (Fig 2b). Immunohistochemistry revealed strongly positive smooth muscle actin expression, while CD-31, EMA, CK, calretinin, and S-100 were all negative. These findings were consistent with a diagnosis of pulmonary artery sarcoma (PAS). Echocardiogram revealed heterogeneous masses inside the main 


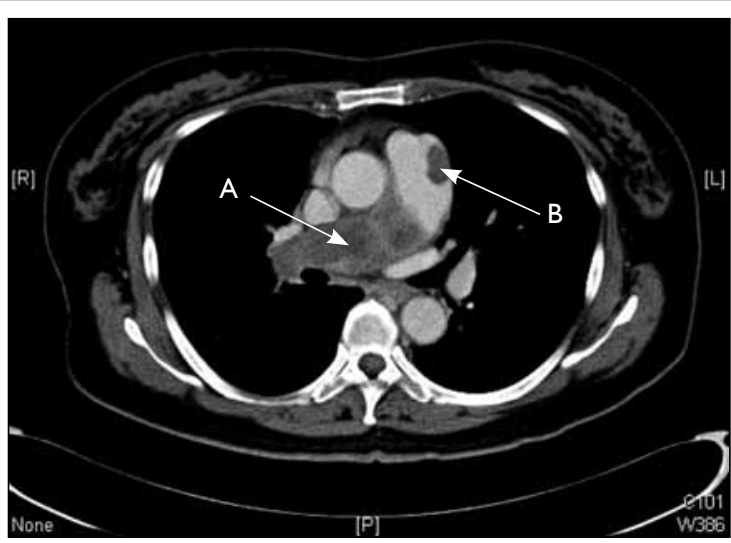

FIG I. Thoracic computed tomography revealing a contrastenhancing mass $(A)$ with low non-homogeneous attenuation filling the right pulmonary artery with extension into the mediastinum. Another mass was also found in the pulmonary trunk (B)
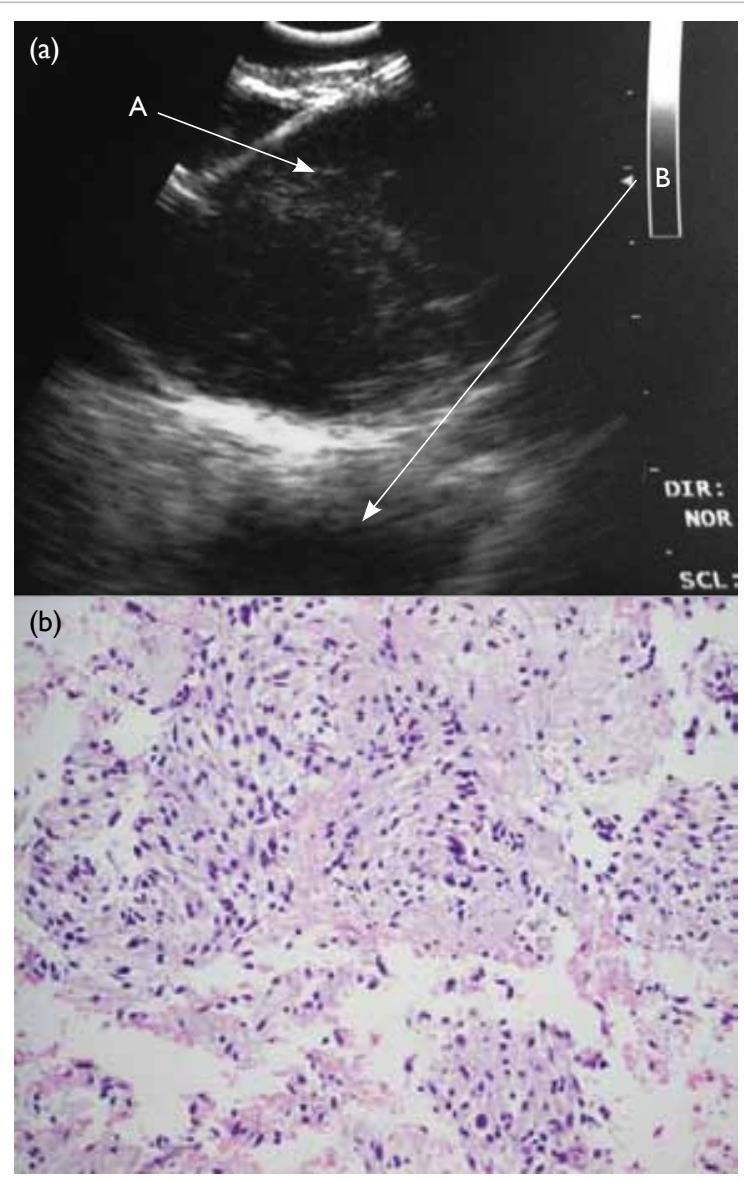

FIG 2. (a) An ultrasonographic view of endobronchial ultrasound-guided transbronchial needle aspiration (TBNA) of a heterogeneous mass at the lower end of trachea (A:

22G TBNA needle; B: outer boundary of the tumour mass).

(b) Spindle cell proliferation in myxoid stroma; tumour cells show mild nuclear pleomorphism (H\&E, x 200)

pulmonary trunk, and between the aorta and the anterior aspect of left atrium, with no evidence of valvular dysfunction or pulmonary hypertension.
Due to the presence of extensive disease and central location of the tumour, aggressive surgery was not considered appropriate by cardiothoracic surgeons. The patient declined palliative chemotherapy or radiotherapy and defaulted from follow-up.

\section{Discussion}

Pulmonary artery sarcoma is a rare condition that was first reported in 1923 from an autopsy. ${ }^{1}$ Apart from a few case series and reviews, ${ }^{2-6}$ most cases were only isolated case reports. Although it is sometimes divided into intimal and mural types, and further histological subtypes, the adherent nature of the tumour and the frequent lack of tissue differentiation sometimes do not allow such classifications, such as in our patient. As both the clinical and radiological features can resemble closely that of pulmonary embolism (PE), some reported cases were initially treated with anticoagulation therapy but without response.,4 A definite diagnosis is usually delayed and made either at autopsy or intra-operatively with frozen sections. ${ }^{5}$ While dyspnoea, cough, and chest pain are the commonest presenting symptoms, ${ }^{2-5}$ haemoptysis has also been reported. ${ }^{3,7}$ Lung involvement is commonly found, ${ }^{3,4}$ although the lung lesion in our patient could represent either tumour deposit or infarct, and both could have led to haemoptysis. On CT scan, the characteristics of PAS often mimic PE. Other features that support a diagnosis of PAS include a low-attenuation defect filling the whole lumen of main or proximal PA and extravascular extension, ${ }^{6}$ both of which were present in our patient. Considering the CT scan findings, along with the absence of clinical features and risk factors of vascular thrombosis, malignancy was suspected in our patient. Apart from CT scan, both magnetic resonance imaging (MRI; with gadolinium 
contrast enhancement) ${ }^{4}$ and fluorodeoxyglucosepositron emission tomography (FDG-PET) ${ }^{7}$ have been described as useful for the diagnosis of PAS. Imaging like CT and MRI may have enabled us to determine the exact nature of peripheral lung lesion in our patient.

The prognosis of PAS is poor, with a median survival of 1.5 months without any treatment. ${ }^{2}$ If possible, radical resection of the tumour should be considered since median survival has been demonstrated to be significantly better with this option (36.5 \pm 20.2 months) than that with incomplete resection such as tumour debulking and thromboendarterectomy (median survival, $11 \pm 3$ months). ${ }^{4}$ Radical resections are usually major operations that might involve extensive resections and subsequent reconstructions and/ or prosthetic replacement of PA, pneumonectomy or thromboendarterectomy, and often requiring cardiopulmonary bypass. ${ }^{4,5}$ Multimodality treatment including neoadjuvant or adjuvant chemotherapy and radiation, together with surgery, might further improve survival. ${ }^{3,4}$ Owing to the rarity of PAS, currently there are no prospective data to guide the best management practices for PAS. Despite the apparent prognostic superiority of curative resection, an adequate cardiopulmonary reserve for the major surgery and the absence of extensive disease that precludes radical resection would be necessary. In view of the extensive disease, radical tumour resection was not considered in our patient. A definite diagnosis at an early stage would improve the chance of survival of PAS patients. However, in the past, such an opportunity was usually not available unless a patient underwent surgery.

Endobronchial ultrasound-guided transbronchial needle aspiration is a minimally invasive diagnostic procedure. The efficacy and safety of EBUS-TBNA, via the bronchoscopic route, in mediastinal and hilar lymph node staging of lung cancer have been well documented, with high sensitivity, specificity, and diagnostic accuracy of $92.3 \%, 100 \%$ and $98 \%$, respectively. ${ }^{8}$ This treatment modality can offer a more cost-effective and less invasive diagnostic option than mediastinoscopy. ${ }^{9}$ It is also useful for diagnosing central pulmonary lesions adjacent to the bronchus ${ }^{10}$ and non-malignant conditions. ${ }^{11}$ Performed in an endoscopic suite, the procedure involves using a special endoscopic instrument incorporating a $7.5-\mathrm{MHz}$ curvilinear ultrasonic transducer at the tip of a flexible bronchoscope, which allows needle aspiration of target lesions under real-time ultrasound guidance via a 22 -gauge needle. The procedure is very safe and can be performed under local anaesthesia and conscious sedation. ${ }^{8-11}$ Our case is the second report in medical literature which illustrates the potential applicability of EBUS-TBNA in a histological diagnosis for PAS through a non-surgical route. In contrast to our case, in the first report describing two patients in literature, the TBNA involved puncturing of the pulmonary arterial wall in one of the patients undergoing the procedure..$^{12}$ Although PA puncture in EBUS-TBNA has been reported to be safe, ${ }^{13}$ self-limiting intramural haematoma and haemopneumomediastinum have been described after an accidental EBUS-TBNA puncture, ${ }^{14}$ and so, the safety of EBUS-TBNA in the routine differential diagnosis of PAS and pulmonary thromboembolism have been doubted. ${ }^{15}$ In contrast, our patient had suspicious radiological features of PAS and absence of clinical features and risks for PE. We believe using EBUS-TBNA to approach extravascular extension of the lesion in such patients offers a relatively non-invasive means for early diagnosis of PAS. However, the safety of PA punctures in EBUSTBNA needs to be clarified in large studies. It must be remembered that performing EBUS-TBNA in patients with acute $\mathrm{PE}$ and pulmonary hypertension may be associated with a high risk of complications such as respiratory distress and bleeding. ${ }^{15}$ Thus, the routine use of EBUS-TBNA as a tool to distinguish PE from the rarer PAS is not encouraged. While MRI or FDG-PET may be considered before performing invasive diagnostic investigations, ${ }^{4}$ the present report suggests that EBUS-TBNA can potentially provide clinicians a diagnostic alternative to surgical exploration for patients suspected of having PAS.

\section{Conclusion}

Pulmonary artery sarcoma is a rare disease with poor prognosis, which is often diagnosed late and frequently mimics PE. Endobronchial ultrasoundguided transbronchial needle aspiration may be a safe and easy option for the early diagnosis of the condition. Extravascular extension of the lesion on thoracic CT provides a diagnostic clue for PAS, and also offers a safe approach for performing EBUSTBNA.

\section{Declaration}

No conflicts of interest were declared by authors.

\section{References}

1. Mandelstamm M. Uber primare neubildungen des herzens [in German]. Virchows Arch 1923;245:43-54.

2. Krüger I, Borowski A, Horst M, de Vivie ER, Theissen P, Gorss-Fengels W. Symptoms, diagnosis and therapy of primary sarcomas of pulmonary artery. Thorac Cardiovasc Surg 1990;38:91-5.

3. Huo L, Moran CA, Fuller GN, Gladish G, Suster S. Pulmonary artery sarcoma: a clinicopathologic and immunohistochemical study of 12 cases. Am J Clin Pathol 2006;125:419-24.

4. Blackmon SH, Rice DC, Correa AM, et al. Management 
of primary pulmonary artery sarcomas. Ann Thorac Surg 2009;87:977-84.

5. Mayer E, Kriegsmann J, Gaumann A, et al. Surgical treatment of pulmonary artery sarcoma. J Thorac Cardiovasc Surg 2001;121:77-82.

6. Yi CA, Lee KS, Choe YH, Han D, Kwon OJ, Kim S. Computed tomography in pulmonary artery sarcoma: distinguishing features from pulmonary embolic disease. J Comput Assist Tomogr 2004;28:34-9.

7. Tueller C, Fischer Biner R, Minder S, et al. FDG-PET in diagnostic work-up of pulmonary artery sarcomas. Eur Respir J 2010;35:444-6.

8. Yasufuku K, Nakajima T, Motoori K, et al. Comparison of endobronchial ultrasound, positron emission tomography, and $\mathrm{CT}$ for lymph node staging of lung cancer. Chest 2006;130:710-8.

9. Navani N, Lawrence DR, Kolvekar S, et al. Endobronchial ultrasound-guided transbronchial needle aspiration prevents mediastinoscopies in the diagnosis of isolated mediastinal lymphadenopathy: a prospective trial. Am J Respir Crit Care Med 2012;186:255-60.

10. Tournoy KG, Rintoul RC, van Meerbeeck JP, et al. EBUS-
TBNA for the diagnosis of central parenchymal lung lesions not visible at routine bronchoscopy. Lung Cancer 2009;63:45-9.

11. Wong M, Yasufuku K, Nakajima T, et al. Endobronchial ultrasound: new insight for the diagnosis of sarcoidosis. Eur Respir J 2007;29:1182-6.

12. Park JS, Chung JH, Jheon S, et al. EBUS-TBNA in the differential diagnosis of pulmonary artery sarcoma and thromboembolism. Eur Respir J 2011;38:1480-2.

13. Vincent B, Huggins JT, Doelken P, Silvestri G. Successful real-time endobronchial ultrasound-guided transbronchial needle aspiration of a hilar lung mass obtained by traversing the pulmonary artery. J Thorac Oncol 2006;1:362-4.

14. Botana-Rial $M$, Núñez-Delgado $M$, Pallarés-Sanmartín A, et al. Intramural hematoma of the pulmonary artery and hemopneumomediastinum after endobronchial ultrasound-guided transbronchial needle aspiration. Respiration 2012;83:353-6.

15. Montani D, Jaïs X, Sitbon O, Dartevelle P, Simonneau G, Humbert M. EBUS-TBNA in the differential diagnosis of pulmonary artery sarcoma and thromboembolism. Eur Respir J 2012;39:1549-50.

\section{Answers to CME Programme Hong Kong Medical Journal February 2014 issue}

Hong Kong Med J 2014;20:52-8

I. Current management of acute scaphoid fractures: a review
A
1. True
2. False
3. True
4. False
5. True
B
1. True
2. True
3. False
4. True
5. True

Hong Kong Med J 2014;20:59-62

\section{The principle of assessing mental capacity for enduring power of attorney}
A
1. False
2. False
3. True
4. True
5. False
B
1. False
2. True
3. True
4. False
5. True 